\title{
Minimum Clinically Important Differences in Oswestry Disability Index Domains and Their Impact on Adult Spinal Deformity Surgery
}

\author{
Go Yoshida ${ }^{1}$, Tomohiko Hasegawa ${ }^{1}$, Yu Yamato ${ }^{1}$, Sho Kobayashi ${ }^{1}$, Oe Shin ${ }^{1}$, \\ Tomohiro Banno ${ }^{1}$, Yuuki Mihara ${ }^{1}$, Hideyuki Arima ${ }^{1}$, Hiroki Ushirozako ${ }^{1}$, \\ Tatsuya Yasuda ${ }^{2}$, Daisuke Togawa ${ }^{1}$, Yukihiro Matsuyama ${ }^{1}$ \\ ${ }^{1}$ Department of Orthopedic Surgery, Hamamatsu University School of Medicine, Hamamatsu, Japan \\ ${ }^{2}$ Department of Orthopedic Surgery, Hamamatsu Medical Center, Hamamatsu, Japan
}

\begin{abstract}
Study Design: Retrospective study.
Purpose: To calculate the minimum clinically important difference (MCID) for total and individual domains of the Oswestry Disability Index (ODI) and assess score distribution and changes over time in surgically treated adult spinal deformity (ASD) patients.

Overview of Literature: Despite the common use of ODI for assessing ASD, there are no robust studies defining MCID values for this index.

Methods: This study included 240 consecutive ASD patients with a minimum of 2 years of follow-up. We calculated MCID values for total and individual ODI domains using all or part of the Scoliosis Research Society-22R questionnaire as anchors. Using current MCID values, we measured the acquisition rates in patients who acquired MCID at follow-up in both total and individual ODI domains. Differences in pathology, age, and locations of the upper and lower instrumented vertebrae were analyzed.

Results: MCID of the total ODI score was $11 \%$, with an area under the curve of 0.737 . Each domain ranged from 0 to 2 , with 1 being the most common value. In the pain and standing domains, $>60 \%$ of the patients acquired MCID, although acquisition rates of the personal care, lifting, sleep, and sexual activity domains were relatively low (20\%-35\%). Patients with MCID had more radiographic improvement in lumbar lordosis, sagittal vertical axis, and T1 pelvic angle than those without MCID $(p<0.05)$.

Conclusions: To our knowledge, this is the first study to describe MCID of ODI (11\%) after ASD surgery. In the pain and standing domains, most patients acquired MCID although the rates of acquisition of MCID in the personal care, lifting, sleep, and sexual activity domains were low. Spine surgeons should counsel their patients regarding the benefits and setbacks of ASD surgery.
\end{abstract}

Keywords: Adult spinal deformity; Minimum clinically important difference; Oswestry Disability Index; Osteotomy; Disability

\section{Introduction}

Previous reports have suggested that up to $60 \%$ of elderly individuals show evidence of a spinal deformity [1]. The primary issues concerning the treatment of adult spinal deformity (ASD) are disability and pain [2,3]. Traditionally, surgical management for ASD patients offers superior radiographic and health-related quality of life (HRQOL)

\footnotetext{
Received Mar 29, 2018; Revised May 28, 2018; Accepted Jul 3, 2018

Corresponding author: Go Yoshida

Department of Orthopedic Surgery, Hamamatsu University School of Medicine, 1-20-1 Handayama, Higashi-ku, Hamamatsu, Shizuoka, 431-3192, Japan

Tel: +81-534352299, Fax: +81-534352296, E-mail: goy@k6.dion.ne.jp
} 
outcomes compared with nonoperative care [4-6], particularly because improvement in pain relief or the ability to stand may contribute to stabilization at physiological sagittal alignment. Conversely, long-segment fusion negatively influences HRQOL, particularly for personal care or bending movements, such as lifting [7]. However, it is not known how long patients will be incapacitated postoperatively before they reach their preoperative status, which domains of QOL have a better or worse outcome for longtime follow-up, and whether good correction is associated with good clinical results.

The minimum clinically important difference (MCID) score is defined as the minimal change in score on an outcome instrument that coincides with the patient's perception of beneficial change or recovery [8-10]. Establishing MCID for HRQOL will help surgeons and patients understand the true surgical outcome of ASD surgery. Recently, MCID values were analyzed and reported for the pain, appearance, and activity domains as well as the subscore and total score of the Scoliosis Research Society-22R questionnaire (SRS-22R) [11,12] in ASD patients undergoing surgical correction $[10,13]$. Additionally, the Oswestry Disability Index (ODI) is a reliable HRQOL parameter in patients undergoing surgery for ASD. Despite the common use of ODI in assessing ASD, there are no robust studies defining the MCID values for ODI, because the MCID values for ODI in previous studies were obtained from patients with lumbar fusion and adult idiopathic patients $[9,10,14,15]$. There is no study describing the distribution of scores for each domain. The present study calculated MCID for total score and each individual domain of ODI using the anchor-based method and assessed the distribution and changes in scores in surgically treated ASD patients with a minimum follow-up period of 2 years.

\section{Materials and Methods}

\section{Patient selection}

The study protocol was approved by the institutional review board of Hamamatsu University School of Medicine (IRB approval no., 14-306). We retrospectively reviewed consecutive patients who underwent posterior corrective spinal fusion surgery for ASD at Hamamatsu University School of Medicine between March 2010 and April 2015. ASD was defined as the presence of at least one of the following indicators: coronal spinal curvature, $\geq 20^{\circ}$; sagittal vertical axis (SVA), $>5 \mathrm{~cm}$; pelvic tilt, $>25^{\circ}$; or thoracic kyphosis, $>60^{\circ}[16]$. The inclusion criteria were (1) age, $\geq 18$ years; (2) number of fused vertebrae, $\geq 4$ segments; (3) results of HRQOL questionnaire of SRS-22R and ODI, available; (4) standing whole-spine and pelvic radiographs, available; and (5) informed consent, available. The diagnoses were defined as (1) congenital deformity, (2) degenerative kyphosis, (3) degenerative kyphoscoliosis, (4) failed back, (5) idiopathic scoliosis, (6) infectious/spinal caries, (7) neuromuscular/Parkinson's disease, and (8) posttraumatic vertebral fracture. Patient demographics are shown in Table 1.

All patients had standing posteroanterior and lateral full spine radiographs at baseline, 6 months, and 1-, 2-, and 5 -year follow-up (all patients had at least 2 years of follow-

Table 1. Demographic data of 240 consecutive surgically treated adult spinal deformity patients

\begin{tabular}{lccc}
\hline Characteristic & Patients & Male sex & Age (yr) \\
\hline All patients & $240(100.0)$ & $42(17.5)$ & $63.4 \pm 16.3$ \\
\hline Diagnosis & & $3(7.1)$ & $34.0 \pm 14.3$ \\
\hline Congenital & $5(2.1)$ & $7(16.7)$ & $68.5 \pm 10.5$ \\
\hline Degenerative kyphosis & $40(16.7)$ & $11(26.2)$ & $69.7 \pm \pm .0$ \\
\hline Degenerative kyphoscoliosis & $77(32.1)$ & $2(4.8)$ & $68.7 \pm 10.8$ \\
\hline Failed back & $13(5.4)$ & $5(11.9)$ & $34.5 \pm 14.5$ \\
\hline Idiopathic & $36(15.0)$ & $2(4.8)$ & $52.7 \pm 9.3$ \\
\hline Infectious/spinal caries & $3(1.3)$ & $8(19.0)$ & $71.5 \pm 5.2$ \\
\hline Neuromuscular/Parkinson & $25(10.4)$ & $4(9.5)$ & $69.8 \pm 8.4$ \\
\hline Vertebral fracture/post-traumatic & $41(17.1)$ & & \\
\hline
\end{tabular}

Values are presented as number (\%) or mean \pm standard deviation. 
up, and some had 5 years of follow-up). Radiographic measurements included thoracic kyphosis (T5-T12), lumbar lordosis (LL, L1-S1), and SVA. Pelvic parameters included sacral slope, pelvic tilt, pelvic incidence, and T1 pelvic angle (TPA).

\section{Patient-reported outcome measures}

ODI was determined for each patient [17]. ODI has emerged as the most commonly recommended conditionspecific outcome measure for patients with spinal disorder. It has 10 sections, which measure pain intensity, personal care, lifting, walking, sitting, standing, sleeping, social life, sex life, and traveling. For each subclass, scores range from 0 (best measured health) to 5 (worst measured health). The SRS-22R is a scoliosis-specific HRQOL questionnaire with 22 items and five domains: pain, appearance, activity, mental, and satisfaction [11,12]. Each domain score ranges from 1 to 5 , with higher scores indicating better outcomes. In this study, ODI was used as an HRQOL parameter because our previous report using a European multicenter study demonstrated that ODI was the most variable measurement, presenting large differences among domains [7].

\section{Anchor-based minimum clinically important differ- ence calculation for Oswestry Disability Index domains}

Several calculation methods have been used to obtain MCID, such as anchor-based methods (comparing HRQOL scores with another measurement) and distribution-based methods (built on the variability of HRQOL scores). Different calculation methods produce different MCID values. The anchor method is one of the most reliable techniques for acquiring MCID. We used the anchor method described in previous studies $[13,14]$.

We used all or part of the SRS-22R as anchors for the MCID values of the ODI domains. The pain domain of ODI was calculated using items $1,2,8$, and 12 of the SRS22R. Similarly, the anchors for personal care, lifting, sitting (items 5 and 12), walking, standing (items 5 and 18), sleeping (items 12 and 13), sexual activity (items 12 and 14), social activity (items 5 and 9), and traveling (items 18 and 9) were determined separately.

The MCID values for the ODI domains were determined using receiver-operating characteristic curve (ROC) analysis with the anchor scores. The cut-off values for an
ROC correspond to the points of optimal tradeoff between sensitivity and specificity to distinguish 'unchanged' and 'changed.' The accuracy of the ROC was evaluated using the calculated area under the curve (AUC).

Using MCIDs for ODI domains, the distribution and change over time for each subclass were analyzed. Differences in pathology (idiopathic, degenerative, Parkinson, or vertebral fracture), age ( $\leq 64,65-74$, or $\geq 75$ years), location of lower instrumented vertebrae (LIV, above L5 or S1 to the ilium), and location of upper instrumented vertebrae (UIV, above T8 or below T9) were also analyzed.

\section{Statistical analysis}

IBM SPSS Statistics ver. 23.0 (IBM Corp., Armonk, NY, USA), was used for statistical analyses. The ROC was used to determine the change in score of ODI with the smallest difference between sensitivity and specificity to identify MCID. The AUC represents the ability of the chosen MCID value to correctly discriminate between improved and nonimproved patients, with AUC values approaching $0.90-1.00$ considered as excellent, $0.80-0.89$ as good, $0.70-0.79$ as better, $0.60-0.69$ as fair, and $0.50-0.59$ as failed.

Means and standard deviations (SDs) were used to describe continuous variables. Changes from baseline to outcomes at 1,2 , and 5 years were evaluated by the paired $t$-test; group comparisons were conducted by the unpaired $t$-test. All $p$-values less than 0.05 were considered to indicate a statistically significant difference.

\section{Results}

\section{Adult spinal deformity surgery}

A total of 240 consecutive patients were enrolled, with a mean age of 63.4 years (SD, 16.3; range, 18-84 years; 42 men, 198 women). The mean follow-up period was 55 months (range, 24-86 months). Seventy-one patients were followed for at least 5 years. The procedures were the posterior approach in 202 patients and the posterior plus anterior approach in 38 patients. All patients underwent deformity correction surgery with long-segment fusion and osteotomy (134 multiple posterior osteotomies, 55 pedicle subtraction osteotomies, and 51 vertebral column resections). UIVs were located from T2 to L2 (T2: 6, T3: 3, T4: 23, T5: 17, T6: 9, T7: 10, T8: 19, T9: 47, T10: 86, 
Table 2. Pre- and postoperative spinopelvic sagittal alignment in 240 consecutive patients

\begin{tabular}{|c|c|c|c|c|c|}
\hline Variable & Preoperative & $6 \mathrm{mo}$ & $1 \mathrm{yr}$ & $2 y r$ & $p$-value (preoperative-1 yr) \\
\hline Sagittal vertical axis (mm) & $108.3 \pm 90.3$ & $43.8 \pm 50.5$ & $56.8 \pm 62.7$ & $66.2 \pm 68.9$ & $<0.0001$ \\
\hline Pelvic incidence $\left({ }^{\circ}\right)$ & $52.4 \pm 11.9$ & $52.4 \pm 11.4$ & $53.2 \pm 12.3$ & $53.0 \pm 12.3$ & Not significant \\
\hline Pelvic tilt $\left({ }^{\circ}\right)$ & $31.8 \pm 13.7$ & $21.4 \pm 10.0$ & $24.7 \pm 10.4$ & $24.9 \pm 11.1$ & $<0.0001$ \\
\hline Sacral slope $\left({ }^{\circ}\right)$ & $20.5 \pm 13.9$ & $30.4 \pm 9.4$ & $28.8 \pm 10.7$ & $28.6 \pm 10.8$ & 0.0004 \\
\hline Lumbar lordosis $\left({ }^{\circ}\right)$ & $19.1 \pm 26.2$ & $41.9 \pm 13.7$ & $40.6 \pm 15.4$ & $39.9 \pm 17.5$ & $<0.0001$ \\
\hline Thoracic kyphosis $\left({ }^{\circ}\right)$ & $27.5 \pm 21.0$ & $33.8 \pm 12.3$ & $39.2 \pm 14.5$ & $40.9 \pm 17.4$ & $<0.0001$ \\
\hline T1 pelvic angle $\left({ }^{\circ}\right)$ & $35.3 \pm 19.1$ & $19.5 \pm 10.4$ & $23.5 \pm 13.2$ & $24.4 \pm 14.3$ & $<0.0001$ \\
\hline
\end{tabular}

Values are presented as mean \pm standard deviation.

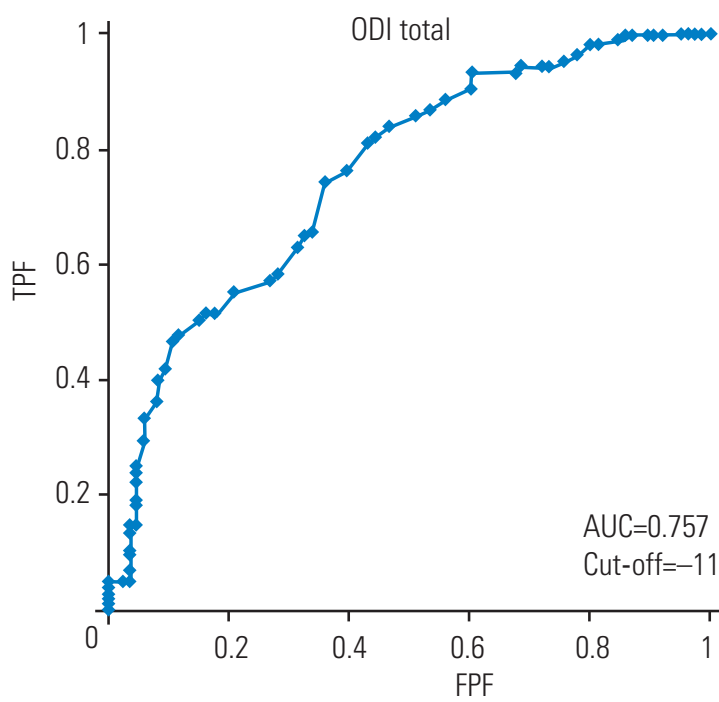

Fig. 1. Receiver-operating characteristic curve analysis indicated minimum clinically important difference of total ODI score was $11 \%$, with AUC of 0.737 , TPF of 0.743 , and FPF of 0.360 . ODI, Oswestry Disability Index; AUC, area under the curve; TPF, true positive fraction (sensitivity); FPF, false positive fraction (1-specificity).

T11: 9, T12: 3, L1: 3, and L2: 5), and LIVs were located from T11 to the ilium (T11: 1, T12: 4, L1: 10, L2: 9, L3: 14, L4: 15, L5: 12, S1: 17, and ilium: 158). All spinopelvic radiographic measurements except pelvic incidence were significantly improved after surgery (Table 2 ).

\section{Minimum clinically important difference for total and individual domain Oswestry Disability Indexes}

Changes in ODI total score 1 year after surgery (1-year postoperative score minus preoperative score) were correlated with changes in SRS-22R total score (1-year postoperative score minus preoperative score) $(r=0.6097$, $p<0.001)$. The mean change in SRS-22R total score was $0.898 \pm 1.009$ (range, -1.41 to 2.64 ). The anchor scores
Table 3. MCID for Oswestry Disability Index total and each domain

\begin{tabular}{lc} 
Domain & MCID \\
\hline Pain & -1 points \\
\hline Personal care & 0 point \\
Lifting & 0 point \\
Walking & -1 points \\
\hline Sitting & -1 points \\
Standing & 2 points \\
\hline Sleep & 0 point \\
\hline Sex activity & -1 points \\
Social activity & -1 points \\
Traveling & -1 points \\
\hline Total & $-11 \%$ \\
\hline
\end{tabular}

MCID, minimum clinically important difference.

were classified as 'worse' ( -1.41 to 0$)$, 'no change' ( 0 to $0.70)$, 'better' ( 0.71 to 1.91$)$, and 'much better' (1.92 to 2.64). The numbers of 'worse,' 'no change,' 'better,' and 'much better' scores were $32,79,98$, and 31 , respectively. MCID of the total ODI score was calculated using ROC analyses to distinguish between 'no change' and 'better' patients. MCID of the total ODI score was 11\% (AUC, 0.737; true positive fraction [or sensitivity, TPF], 0.743; false positive fraction [or 1-specificity, FPF], 0.360) (Fig. 1). The mean sum of items $1,2,8$, and 12 of the SRS-22R was $1.617 \pm 3.186$ (range, -8 to 10 ). The anchor scores were classified as 'much worse' ( -8 to -5$)$, 'worse' $(-4$ to -2$)$, 'no change' ( -1 to 1$)$, 'better' ( 2 to 4$)$, or 'much better' (5 to 10). The numbers of 'much worse,' 'worse,' 'no change,' 'better', and 'much better' scores were 18, 34, 71, 91, and 26 , respectively. MCID of the pain intensity domain was calculated using ROC analyses to distinguish 'no change' and 'better' patients. MCID of the pain domain was -1 


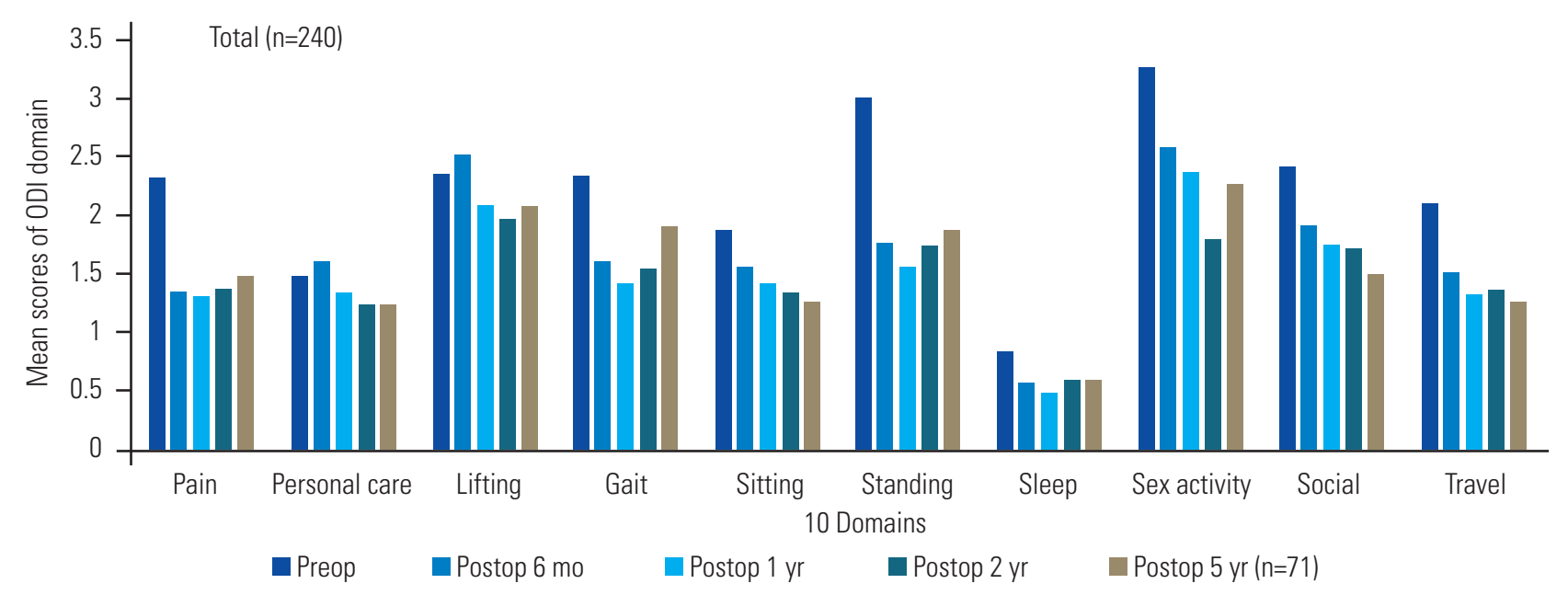

Fig. 2. Mean ODI points of 10 domains at baseline and at 6-month and 1-, 2-, and 5-year follow-up after surgery in 240 consecutive adult spinal deformity patients (only 71 patients reached the 5-year follow-up). ODI, Oswestry Disability Index; Preop, preoperative; Postop, postoperative.

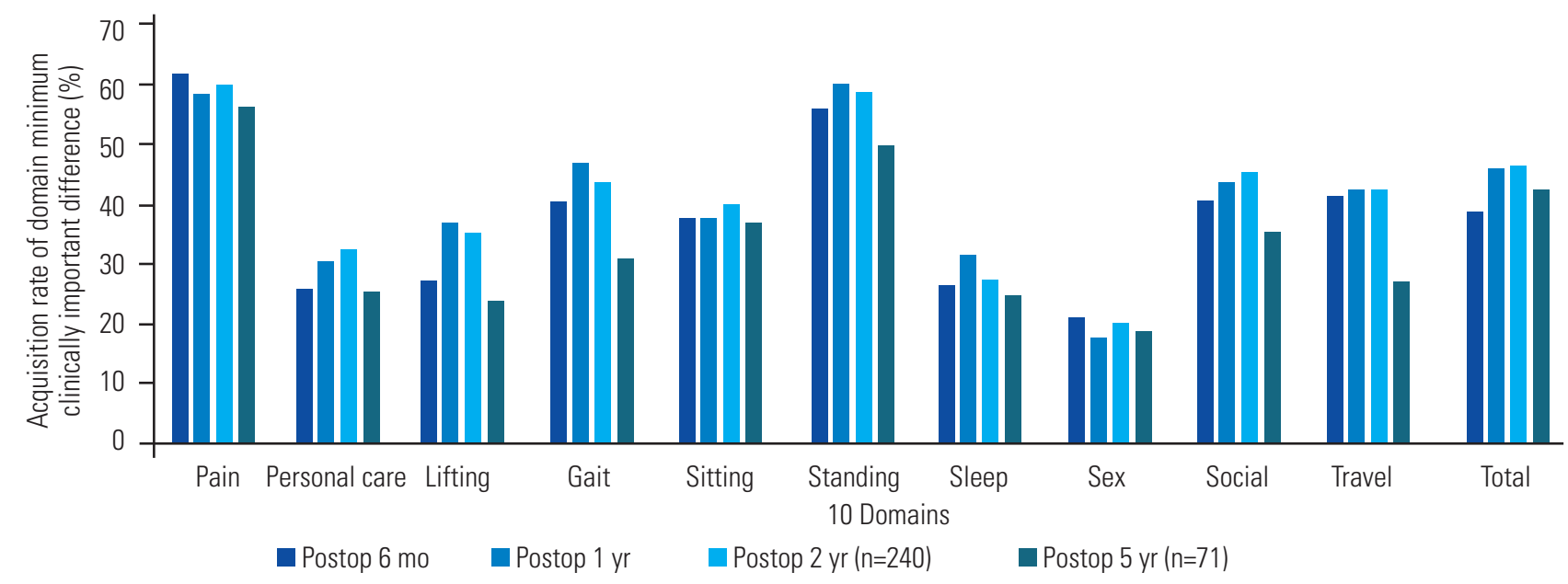

Fig. 3. Acquisition rates of 10 Oswestry Disability Index domains at 6-month and 1-, 2-, and 5-year follow-up after surgery in 240 consecutive adult spinal deformity patients (only 71 patients reached the 5-year follow-up). Preop, preoperative; Postop, postoperative.

(AUC, 0.625; TPF, 0.725; FPF, 0.535). MCIDs of the remaining domains were determined using a similar calculation, as follows: personal care, 0 ; lifting, 0; walking, -1 ; sitting, -1 ; standing, -2 ; sleeping, 0 ; sexual activity, -1 ; social life, -1 ; and traveling, -1 (Table 3).

\section{Distribution and change in Oswestry Disability Index domain over time}

All 240 patients answered questions in nine categories of the ODI questionnaire pre- and postoperatively except for the question on sexual activity domain. The question on sexual activity domain was answered by 61 of the 240 patients (25.4\%). Fig. 2 shows the mean number of ODI domain points at baseline and at 6 months, 1 year, 2 years, and 5 years after surgery. Globally, the sitting, social activity, and traveling domains had good results continuously until 5 years postoperatively. Scores for the personal care and lifting subclasses were worse 6 months after surgery; however, they recovered 1 and 2 years after surgery. At 2-year follow-up, all domains were improved compared with preoperative status; however, pain, gait, and standing domains became worse between 1 and 5 years after surgery. We calculated the acquisition rate as the proportion of patients who acquired MCID at follow-up times in both the total and individual ODI domains:

Acquisition rate $($ total $)=$ number of patients who ac- 
quired MCID (-11)/240×100.

Acquisition rate (nine domains except for the sexual activity domain $)=$ number of patients who acquired MCID $(-1) / 240 \times 100$.

Acquisition rate (sexual activity domain)=number of patients who acquired MCID $(-1) / 61 \times 100$.

In total, the acquisition rates at 6-month, 1-year, 2-year, and 5-year follow-up were 38.8\% (93/240), 46.3\% (111/240), 46.7\% (112/240), and 42.3\% (30/71). It improved 1 year postoperatively, was maintained until 2-year follow-up, and decreased at 5-year follow-up. Fig. 3 shows that the difference in each domain was approximately $20 \%-60 \%$. The acquisition rates for the pain and standing domains were very high (approximately 60\%). Conversely, those for personal care, lifting, sleep, and sexual activity were relatively low (approximately 30\%). Most domain acquisition rates increased up to the 2-year follow-up and decreased at the 5-year follow-up.

\section{Radiographic characteristics of patients with or with- out minimum clinically important difference in total Oswestry Disability Index}

Table 4 shows the mean radiographic parameters of patients with and without MCID. There were significant differences between the pre- and postoperative sagittal parameters in both groups. Two years postoperatively, there was no significant difference in sagittal parameters between patients with and without MCID; however, there were significant differences in improvement of LL, SVA, and TPA between patients with and without MCID.

\section{Minimum clinically important difference acquisition rates according to pathology, age, upper- and lower- instrumented vertebrae}

A total of 77, 36, 25, and 41 patients had degenerative kyphoscoliosis, idiopathic, Parkinson, and vertebral fracture pathologies, respectively. The pre- and postoperative total ODI scores for idiopathic scoliosis were lower than those for other pathologies; therefore, the acquisition rate for idiopathic scoliosis had the lowest value. Patients were divided into three age groups: $\leq 64,65-74$, and $\geq 75$ years. The pre- and postoperative total ODI scores increased with increasing age. However, the acquisition rate for the older group was higher than those for the other age groups. Patients with UIV above T8 were younger and had lower preoperative total ODI scores than those with UIV below T9. Conversely, patients with UIV above T8 had higher postoperative total ODI scores than those with UIV below T9. Therefore, the acquisition rate of those with UIV above T8 was lower than that of patients with UIV below T9.

Patients with LIV above L5 were younger and had lower pre- and postoperative total ODI scores than those with LIV to the pelvis. Therefore, the acquisition rate of patients with LIV above L5 was lower than those of patients with LIV to the pelvis (Table 5).

Table 4. Radiographic characteristics of the patient with- and without MCID in total Oswestry Disability Index

\begin{tabular}{|c|c|c|c|c|c|c|c|c|}
\hline \multirow{2}{*}{ Variable } & \multicolumn{3}{|c|}{ Patients with MCID } & \multicolumn{3}{|c|}{ Patients without MCID } & \multirow{2}{*}{$p$-value ${ }^{\mathrm{a})}$} & \multirow{2}{*}{$p$-value ${ }^{b}$} \\
\hline & Preop & Postop 2 yr & Improve & Preop & Postop 2 yr & Improve & & \\
\hline Lumbar lordosis $\left({ }^{\circ}\right)$ & $14.0 \pm 22.8$ & $41.0 \pm 17.7$ & $26.8 \pm 9.7$ & $23.6 \pm 28.3$ & $39.0 \pm 17.4$ & $16.0 \pm 8.9$ & NS & $<0.05$ \\
\hline Thoracic kyphosis $\left({ }^{\circ}\right)$ & $23.6 \pm 19.3$ & $38.6 \pm 13.7$ & $14.9 \pm 8.0$ & $31.0 \pm 21.9$ & $42.7 \pm 20.0$ & $11.7 \pm 8.1$ & NS & NS \\
\hline Sacral slope $\left({ }^{\circ}\right)$ & $19.7 \pm 12.2$ & $28.9 \pm 10.8$ & $9.1 \pm 5.6$ & $21.2 \pm 15.2$ & $28.3 \pm 10.8$ & $7.1 \pm 5.4$ & NS & NS \\
\hline Pelvic incidence $\left({ }^{\circ}\right)$ & $53.1 \pm 11.9$ & $53.1 \pm 12.3$ & $0.1 \pm 1.1$ & $51.9 \pm 12.1$ & $52.9 \pm 12.3$ & $0.9 \pm 1.0$ & NS & NS \\
\hline Pelvic tilt $\left({ }^{\circ}\right)$ & $33.3 \pm 11.8$ & $25.3 \pm 10.3$ & $8.1 \pm 3.8$ & $30.5 \pm 15.2$ & $24.6 \pm 11.8$ & $6.0 \pm 3.1$ & NS & NS \\
\hline Sagittal vertical axis (mm) & $127.5 \pm 88.1$ & $58.5 \pm 58.3$ & $69.1 \pm 74.0$ & $91.6 \pm 89.5$ & $71.9 \pm 76.3$ & $19.9 \pm 24.5$ & NS & $<0.05$ \\
\hline T1 pelvic angle $\left(^{\circ}\right)$ & $37.6 \pm 16.2$ & $23.4 \pm 13.1$ & $14.2 \pm 9.0$ & $33.2 \pm 21.2$ & $25.1 \pm 15.2$ & $8.1 \pm 5.5$ & NS & $<0.05$ \\
\hline
\end{tabular}

Values are presented as mean \pm standard deviation. Improve value indicates postoperative minus preoperative. MCID, minimum clinically important difference; Preop, preoperative; Postop, postoperative; NS, not significant.

${ }^{a}$ The examination was performed between the patients with and without MCID in postoperative 2 years. ${ }^{b}$ The examination was performed between the patients with and without MCID in improve value. 
Table 5. Minimum clinically important difference acquisition rate in different pathology, age, LIV and UIV

\begin{tabular}{|c|c|c|c|c|}
\hline Variable & Mean age (yr) & Preop ODI & Postop ODI & Acquisition rate \\
\hline \multicolumn{5}{|l|}{ Pathology } \\
\hline Degenerative kyphoscoliosis & $69.7 \pm 7.0$ & $45.6 \pm 17.4$ & $32.3 \pm 20.6$ & $49.4(38 / 77)$ \\
\hline Idiopathic & $34.5 \pm 14.5$ & $18.9 \pm 15.6$ & $12.6 \pm 12.8$ & $37.5(13 / 36)$ \\
\hline Parkinson & $71.5 \pm 5.2$ & $52.5 \pm 20.4$ & $43.3 \pm 21.1$ & $48.0(12 / 25)$ \\
\hline Vertebral fracture & $69.8 \pm 8.4$ & $58.8 \pm 19.0$ & $42.3 \pm 23.5$ & $48.8(20 / 41)$ \\
\hline \multicolumn{5}{|l|}{ Age groups (yr) } \\
\hline$\leq 64$ & $45.2 \pm 16.0$ & $30.2 \pm 21.1$ & $21.9 \pm 19.0$ & $42.5(34 / 80)$ \\
\hline $65-74$ & $69.5 \pm 2.9$ & $47.6 \pm 16.8$ & $36.7 \pm 22.4$ & $45.5(45 / 99)$ \\
\hline$\geq 75$ & $77.4 \pm 2.3$ & $51.8 \pm 18.2$ & $36.9 \pm 20.2$ & $54.1(33 / 61)$ \\
\hline \multicolumn{5}{|l|}{ LIV } \\
\hline Above L5 & $49.8 \pm 22.8$ & $26.2 \pm 24.3$ & $26.8 \pm 25.5$ & $33.8(22 / 65)$ \\
\hline S1 to ilium & $68.6 \pm 8.9$ & $46.9 \pm 17.4$ & $32.6 \pm 20.0$ & $51.4(90 / 175)$ \\
\hline \multicolumn{5}{|l|}{ UIV } \\
\hline Above T8 & $59.5 \pm 19.8$ & $37.1 \pm 24.0$ & $33.0 \pm 24.4$ & $36.0(31 / 86)$ \\
\hline Below T9 & $65.5 \pm 13.6$ & $45.3 \pm 18.4$ & $30.9 \pm 20.2$ & $52.5(81 / 154)$ \\
\hline
\end{tabular}

Values are presented as mean \pm standard deviation or \% (number/total number).

LIV, lower instrumented vertebrae; UIV, upper instrumented vertebrae; Preop, preoperative; Postop, postoperative; ODI, Oswestry Disability Index.

\section{Discussion}

HRQOL is important for evaluating the efficacy of surgical treatment. Several outcome-related studies have established that surgical treatment of ASD patients is likely to result in threshold improvement and simultaneous improvements across multiple SF-36, ODI, and SRS-22 measures $[1,4-6,18]$. ODI is a generic HRQOL questionnaire that is extensively used to evaluate ASD.

In general, the mean change in score from before to after surgery did not correlate with individual outcomes, such as patient satisfaction or disappointment. MCID is commonly used to quantify a threshold of improvement that is clinically relevant to the individual patient for various outcome measures [8-10,19,20]. Additionally, the MCID value may help researchers understand the distribution score for the improvement rate among different subclasses or domains. The present study indicated that the mean ODI domain scores were different from the acquisition rates of the ODI domain MCID (Figs. 2, 3). Berven et al. [10] reported that MCID after ASD surgery was 0.59 for the SRS-22R pain domain, 0.8 for the appearance domain, 0.38 for the activity domain, and 0.42 for the mental domain. Crawford et al. [13] found that MCID after ASD surgery was 0.40 for the pain domain, 1.23 for the appearance domain, and 0.60 for the activity domain and the subscore was 0.43 . These values varied due to differences in patient samples and calculation methods. Conversely, there have been few analyses of MCID values of ODI after ASD surgery. MCID values of the total ODI have been reported as $8 \%$ to $30 \%$, according to the target disease and intervention [21-25]. The diseases ranged from low back pain to failed back syndrome, and the interventions varied from physical therapy to complex spinal surgery. The MCID values of ODI after lumbar fusion were 10 to $12.8[9,14,15]$. MCID in ASD surgery was reported as 15.0 at an international meeting [10], and most authors have adopted this value as MCID after ASD surgery [26-28].

We calculated MCID values for total and individual domains of ODI after ASD surgery to analyze the distribution of scores. We determined that MCID for total ODI was $11 \%$ and ODI values for individual domains were 0 to 2 and were mostly 1 by the anchor-based method using SRS-22. MCID value after ASD surgery in the present series is similar to the results of previous studies with data obtained after lumbar fusion $[9,14,15]$.

Numerous studies have established that ASD surgery results in improved HRQOL and decreased disability. However, few studies have directly investigated improve- 
ment in ODI subclasses. A previous study demonstrated the advantages and disadvantages after ASD surgery [7]; two disadvantages were observed in the HRQOL subclasses of personal care and lifting, which were correlated with sagittal modifiers of the SRS-Schwab classification [16]. Similarly, our study found that personal care and lifting were negatively affected until 6 months after ASD surgery, and only $30 \%$ of patients reached MCID for these two domains. Lifting was worse after ASD surgery. Lifting requires movement and muscle strength in the paraspinal muscles and the hip and knee joint extensors. Nevertheless, considering the patient's pathology and LIV, spinal fusion strongly influences the paraspinal muscles. When the patients were divided based on UIV or LIV, those with a higher UIV and lower LIV had much worse outcomes than those with shorter fusion areas. For elderly ASD patients with osteoporosis, the decision to end fusion remains controversial, particularly if the L5-S1 disc is healthy. A few studies have compared HRQOL with LIV at L5 versus extension to the sacrum. There were no significant differences between the groups in functional outcome measures based on ODI, although fusion to the sacrum required an increased number of procedures and was associated with a higher frequency of complications [29].

These negative effects after ASD surgery may be greater for lifestyle activities that involve bending the trunk, performing on the floor, getting up from the floor, squatting on the toilet, and performing manual agricultural work. Therefore, limitations after ASD surgery should be tracked over time to better inform patients. In most countries, personal care is one of the most important daily activities for people of all ages. Tasks involved in personal care include dressing, bathing, and toilet functions. Sleep and sexual activity showed little improvement in the present study. Conversely, the pain relief and standing domains were positively affected after ASD surgery, with a rate of MCID acquisition of almost $60 \%$. Furthermore, patients who achieved MCID had more radiographic improvement in LL, SVA, and TPA than those who did not achieve MCID. Interestingly, the acquisition rates were much higher for patients with degenerative pathology, elderly patients (age $\geq 75$ years), and patients with LIV to the pelvis than those for patients with idiopathic pathology, younger patients (age $\leq 64$ years), and patients with LIV stopped at L5. This is because pre- and postoperative ODI scores were much lower for patients with idiopathic pathology, younger patients, and patients with LIV stopped at L5 than for patients with degenerative pathology, elderly patients, and patients with LIV to the pelvis. Thus, MCID is affected by the baseline. The lower the baseline, the higher the acquisition rate, and the higher the baseline, the lower the acquisition rate. In other words, MCID has a limitation that it is influenced by the baseline data, such as preoperative ODI scores.

This study had several limitations. First, it was retrospective in nature, although the data used were prospectively collected. Second, MCID was analyzed by an anchor-based method by SRS-22. Previous studies have reported various MCID values for HRQOL after ASD surgery using anchor-based methods $[9,13-15,19]$; therefore, we also used that method, although the exact MCID values varied. Third, our MCID values were based on Japanese ASD patients with different pathologies, alignments, and HRQOL compared with other those in other nations. Patient ethnicity should be considered when evaluating HRQOL measures and radiographic parameters [30,31]. Japan has the highest percentage of elderly people of all countries; therefore, these results should help in understanding age-related spinal deformity in other countries. Finally, this study was performed in a single center with a limited number of spinal deformity surgeons. However, this may be considered as an advantage because of the unified surgical indications, strategies, and procedures. Recent multicenter studies were superior in terms of standardized data collection and generalizability of the results; however, these results may be influenced by different surgical strategies and institutional experiences. In the future, MCID of HRQOL measures should be studied in prospective multicenter studies with standardized data collection and a generalized surgical strategy.

\section{Conclusions}

This is the first study to describe MCID of ODI in the total and individual domains after ASD surgery. Total MCID of ODI was $11 \%$ in our survey and was mostly 1 for each individual domain. In the pain and standing domains, $>60 \%$ of patients acquired MCID, although the acquisition rates for the personal care, lifting, sleep, and sexual activity domains were relatively low. Spine surgeons should counsel their patients regarding the benefits and setbacks of ASD surgery. 


\section{Conflict of Interest}

No potential conflict of interest relevant to this article was reported.

\section{References}

1. Schwab F, Dubey A, Gamez L, et al. Adult scoliosis: prevalence, SF-36, and nutritional parameters in an elderly volunteer population. Spine (Phila Pa 1976) 2005;30:1082-5.

2. Schwab FJ, Blondel B, Bess S, et al. Radiographical spinopelvic parameters and disability in the setting of adult spinal deformity: a prospective multicenter analysis. Spine (Phila Pa 1976) 2013;38:E803-12.

3. Glassman SD, Berven S, Bridwell K, Horton W, Dimar JR. Correlation of radiographic parameters and clinical symptoms in adult scoliosis. Spine (Phila Pa 1976) 2005;30:682-8.

4. Li G, Passias P, Kozanek M, et al. Adult scoliosis in patients over sixty-five years of age: outcomes of operative versus nonoperative treatment at a minimum two-year follow-up. Spine (Phila Pa 1976) 2009;34:2165-70.

5. Smith JS, Shaffrey CI, Glassman SD, et al. Riskbenefit assessment of surgery for adult scoliosis: an analysis based on patient age. Spine (Phila Pa 1976) 2011;36:817-24.

6. Bridwell KH, Glassman S, Horton W, et al. Does treatment (nonoperative and operative) improve the two-year quality of life in patients with adult symptomatic lumbar scoliosis: a prospective multicenter evidence-based medicine study. Spine (Phila Pa 1976) 2009;34:2171-8.

7. Yoshida G, Boissiere L, Larrieu D, et al. Advantages and disadvantages of adult spinal deformity surgery and its impact on health-related quality of life. Spine (Phila Pa 1976) 2017;42:411-9.

8. Carragee EJ, Cheng I. Minimum acceptable outcomes after lumbar spinal fusion. Spine J 2010;10:313-20.

9. Glassman SD, Copay AG, Berven SH, Polly DW, Subach BR, Carreon LY. Defining substantial clinical benefit following lumbar spine arthrodesis. J Bone Joint Surg Am 2008;90:1839-47.

10. Berven S, Deviren V, Demir-Deviren S, Hu S, Bradford D. Minimal clinically important difference in adult spinal deformity: how much change is significant. Proceedings of the International Meeting for
Advanced Spine Techniques (IMAST); 2005 Jul 7-9; Banff, Canada. Milwaukee (WI): Scoliosis Research Society; 2005.

11. Berven S, Deviren V, Demir-Deviren S, Hu SS, Bradford DS. Studies in the modified Scoliosis Research Society Outcomes Instrument in adults: validation, reliability, and discriminatory capacity. Spine (Phila Pa 1976) 2003;28:2164-9.

12. Bridwell KH, Cats-Baril W, Harrast J, et al. The validity of the SRS-22 instrument in an adult spinal deformity population compared with the Oswestry and SF-12: a study of response distribution, concurrent validity, internal consistency, and reliability. Spine (Phila Pa 1976) 2005;30:455-61.

13. Crawford $\mathrm{CH}$ 3rd, Glassman SD, Bridwell KH, Berven SH, Carreon LY. The minimum clinically important difference in SRS-22R total score, appearance, activity and pain domains after surgical treatment of adult spinal deformity. Spine (Phila Pa 1976) 2015;40:377-81.

14. Copay AG, Glassman SD, Subach BR, Berven S, Schuler TC, Carreon LY. Minimum clinically important difference in lumbar spine surgery patients: a choice of methods using the Oswestry Disability Index, Medical Outcomes Study questionnaire Short Form 36, and pain scales. Spine J 2008;8:968-74.

15. Hagg O, Fritzell P, Nordwall A; Swedish Lumbar Spine Study Group. The clinical importance of changes in outcome scores after treatment for chronic low back pain. Eur Spine J 2003;12:12-20.

16. Schwab F, Ungar B, Blondel B, et al. Scoliosis Research Society-Schwab adult spinal deformity classification: a validation study. Spine (Phila Pa 1976) 2012;37:1077-82.

17. Fairbank JC, Pynsent PB. The Oswestry Disability Index. Spine (Phila Pa 1976) 2000;25:2940-52.

18. Ware JE Jr, Sherbourne CD. The MOS 36-item shortform health survey (SF-36): I. conceptual framework and item selection. Med Care 1992;30:473-83.

19. Carreon LY, Sanders JO, Diab M, et al. The minimum clinically important difference in Scoliosis Research Society-22 appearance, activity, and pain domains after surgical correction of adolescent idiopathic scoliosis. Spine (Phila Pa 1976) 2010;35:2079-83.

20. Liu S, Schwab F, Smith JS, et al. Likelihood of reaching minimal clinically important difference in adult spinal deformity: a comparison of operative and non- 
operative treatment. Ochsner J 2014;14:67-77.

21. Fritz JM, Hebert J, Koppenhaver S, Parent E. Beyond minimally important change: defining a successful outcome of physical therapy for patients with low back pain. Spine (Phila Pa 1976) 2009;34:2803-9.

22. Gatchel RJ, Mayer TG. Testing minimal clinically important difference: consensus or conundrum? Spine J 2010;10:321-7.

23. Ostelo RW, Deyo RA, Stratford P, et al. Interpreting change scores for pain and functional status in low back pain: towards international consensus regarding minimal important change. Spine (Phila Pa 1976) 2008;33:90-4.

24. Park KB, Shin JS, Lee J, et al. Minimum clinically important difference and substantial clinical benefit in pain, functional, and quality of life scales in failed back surgery syndrome patients. Spine (Phila $\mathrm{Pa}$ 1976) 2017;42:E474-81.

25. Van der Roer N, Ostelo RW, Bekkering GE, van Tulder MW, de Vet HC. Minimal clinically important change for pain intensity, functional status, and general health status in patients with nonspecific low back pain. Spine (Phila Pa 1976) 2006;31:578-82.

26. Blondel B, Schwab F, Ungar B, et al. Impact of magnitude and percentage of global sagittal plane correction on health-related quality of life at 2-years follow- up. Neurosurgery 2012;71:341-8.

27. Scheer JK, Lafage V, Smith JS, et al. Impact of age on the likelihood of reaching a minimum clinically important difference in 374 three-column spinal osteotomies: clinical article. J Neurosurg Spine 2014;20:306-12.

28. Fakurnejad S, Scheer JK, Lafage V, et al. The likelihood of reaching minimum clinically important difference and substantial clinical benefit at 2 years following a 3-column osteotomy: analysis of 140 patients. J Neurosurg Spine 2015;23:340-8.

29. Cho KJ, Suk SI, Park SR, et al. Arthrodesis to L5 versus S1 in long instrumentation and fusion for degenerative lumbar scoliosis. Eur Spine J 2009;18:531-7.

30. Ames C, Gammal I, Matsumoto M, et al. Geographic and ethnic variations in radiographic disability thresholds: analysis of North American and Japanese operative adult spinal deformity populations. Neurosurgery 2016;78:793-801.

31. Diebo BG, Gammal I, Ha Y, et al. Role of ethnicity in alignment compensation: propensity matched analysis of differential compensatory mechanism recruitment patterns for sagittal malalignment in 288 ASD patients from Japan, Korea, and United States. Spine (Phila Pa 1976) 2017;42:E234-40. 